\title{
O ALICIAMENTO SEXUAL DE MENORES NA INTERNET: CONTRIBUTOS PARA O SEU CONHECIMENTO E PREVENÇÃO
}

\author{
Cristiana Barbosa \\ Faculdade de Psicologia e Ciências da Educação da Universidade do Porto (FPCEUP) \\ Gabinete de Estudos e de Atendimento a Agressores e Vítimas (GEAV) \\ cristiana.psi@outlook.pt \\ Celina Manita
}

Faculdade de Psicologia e Ciências da Educação da Universidade do Porto (FPCEUP) / Gabinete de Estudos e de Atendimento a Agressores e Vítimas (GEAV)

celina@fpce.up.pt

Fecha de Recepción: 16 Junio 2019

Fecha de Admisión: 25 Septiembre 2019

\begin{abstract}
RESUMO
A crescente utilização da Internet e de redes sociais por crianças e jovens tem vindo a criar condições para 0 desenvolvimento de novas modalidades de grooming sexual, um processo facilitador da perpetração de crimes sexuais, na medida em que permite um acesso facilitado a um elevado número de potenciais vítimas, aumenta as oportunidades para o estabelecimento de contactos, quer online quer diretos, e facilita a identificação de vulnerabilidades nos menores. Este é um fenómeno em expansão, tornando-se urgente conhecê-lo, de forma a desenvolver estratégias eficazes de promoção da segurança das crianças e jovens na Internet e evitar danos decorrentes desta forma de abuso. 0 presente estudo recorreu a uma metodologia qualitativa, utilizando como métodos de recoIha de dados a entrevista semiestruturada e a análise documental, submetidos a uma análise de conteúdo, com construção de uma grelha de análise categorial do tipo semântico. A amostra englobou 4 ofensores do sexo masculino, 2 entrevistados face-a-face e 2 envolvidos em diálogos sexualizados mantidos online com menores de idade, e 1 vítima do sexo feminino, também entrevistada. Os resultados permitiram distinguir duas abordagens distintas dos menores - uma muito direta, com a introdução quase imediata de temáticas sexuais, e outra em que o sujeito adota um conjunto de estratégias que têm por base a criação e manutenção de um vínculo relacional, de acordo com os mecanismos considerados centrais no grooming. Enquanto na interação mais direta 0 ofensor parece aproveitar-se da curiosidade sexual típica do período de desenvolvimento, na segunda abordagem o sujeito recorre ao vínculo relacional para conseguir o envolvimento dos menores e evitar a revelação dos abusos. Apesar de o grooming surgir na literatura como um componente central das
\end{abstract}




\section{O ALICIAMENTO SEXUAL DE MENORES NA INTERNET: CONTRIBUTOS PARA O SEU CONHECIMENTO E PREVENÇÃO}

interações sexuais online com menores, constatamos neste estudo que ele não é necessário para a efetiva concretização de diferentes tipos de abuso.

Palavras-chave: abuso sexual; grooming sexual; internet; grooming sexual online; estratégias.

\section{ABSTRACT}

Online Sexual Grooming of Minors: Contributions to its Knowledge and Prevention. The growing use of the Internet and of social media by children and young people has spurred the conditions for the development of sexual grooming, a process that facilitates the perpetration of sexual crimes, as it allows an easier access to a high number of potential victims, increases the opportunities to perpetrate both online and personal contact with minors, and facilitates the identification of vulnerabilities in the victims. This is a growing phenomenon and it is urgent to know it, in order to develop effective strategies to promote the safety of children and young people on the Internet and to prevent the harm resulting from this type of abuse. In order to do it we developed a qualitative study, using semi-structured interviews and document analysis, submitted to a content analysis (a categorical semantic analysis). The sample consisted of 4 male offenders, 2 face-to-face interviewees and 2 involved in online sexualized dialogues with minors, and 1 female victim, who was also interviewed. The results allowed to distinguish two distinct approaches, one very direct, with the almost immediate introduction of sexual themes, and another approach in which the subject adopt a set of strategies that are based on the creation and maintenance of a relational bond, being consistent with what is considered the core strategies of the grooming process. While in the most direct approach the offender seems to take advantage of the typical sexual curiosity of the developmental period, in the second approach the subject uses a relational bond to get the involvement of the minor and avoid disclosure. Although grooming appears in the literature as a major component of online sexual interactions with minors, we found in our research that grooming is not necessary for achieving sexual contacts or child sexual abuse.

Keywords: child sexual abuse; sexual grooming; internet; grooming online; strategies.

\section{INTRODUÇÃO}

As gerações juvenis estão cada vez mais presentes no mundo digital, estando vulneráveis aos riscos que a conectividade comporta, tais como novas formas de exploração sexual e vitimização (UNICEF, 2017). A Internet veio alterar o processo de grooming sexual tradicional, referente às ações levadas a cabo por um ofensor para criar as condições necessárias para cometer uma ofensa sexual contra uma criança sem ser descoberto (Craven, Brown, \& Gilchrist, 2006; McAlinden, 2006). Para tal, existe uma manipulação estratégica da vítima e da respetiva família e comunidade, com recurso à construção de relações de confiança com estes que tornam menos provável a deteção dos abusos ou a denúncia por parte da criança e a perceção do ofensor como uma ameaça por parte dos responsáveis (McAlinden, 2006; Winters \& Jeglic, 2016).

A nova variante designada por grooming sexual online carateriza-se pela utilização das novas tecnologias para aliciar a criança, sendo estabelecida uma interação de cariz sexual nas plataformas de comunicação via Internet, que pode cingir-se a estas ou levar ao contacto direto (Branca, Grangeia, \& Cruz, 2016; Davidson et al., 2011; Webster et al., 2012). No mundo virtual, o ofensor não necessita de manipular os pais ou outros adultos significativos para alcançar a criança, ultrapassando os limites do controlo social, o que facilita a velocidade a que o processo ocorre (Black, Wollis, Woodworth, \& Hancock, 2015; Webster et al., 2012), contribuindo também para tal a não existência de fronteiras geográficas e 0 facto de a proximidade física com as vítimas não ser um prérequisito (Berson, 2003; Webster et al., 2012). 
Com recurso às ferramentas digitais, o ofensor pode encetar contactos em diferentes cenários e com várias vítimas simultaneamente, desenvolvendo interações diárias e privadas que, de outro modo, só seriam possíveis se fosse o cuidador do menor (Branca et al., 2016; Davidson et al., 2011). 0 mundo digital permite ainda que 0 ofensor possa realizar alterações nas suas características, podendo a identidade com a qual se apresenta diferir da sua identidade real (Webster et al., 2012).

0 estabelecimento de contactos com os menores pode ser aleatório, ou realizado através da leitura dos perfis online e do contacto com comunidades virtuais de jovens, onde são procuradas referências a conteúdos sexuais ou indicadores de vulnerabilidade, por serem consideradas reveladoras, respetivamente, de desinibição face às temáticas sexuais e de maior facilidade de manipulação/submissão (Malesky, 2007).

A literatura tem demonstrado que a abordagem dos menores pode assumir contornos diferentes. Enquanto alguns indivíduos seguem o processo de socialização característico da definição mais tradicional do grooming, criando primeiramente um vínculo com a criança, outros são mais diretos, ocorrendo uma introdução imediata das temáticas sexuais (Kloess, Hamilton-Giachritsis, \& Beech, 2017; Webster et al., 2012; Wolak, Finkelhor, \& Mitchell, 2004).

Os adolescentes são dos maiores utilizadores do mundo digital, sendo os mais afetados por esta nova forma de vitimização (Wachs, Wolf, \& Pan, 2012). As organizações internacionais (UNICEF, 2017) têm alertado para a necessidade de compreender o fenómeno do abuso por meio de tecnologias de comunicação e informação, de forma a desenvolver políticas e medidas de prevenção.

\section{OBJETIVOS DA INVESTIGAÇÃO}

0 objetivo geral deste estudo consiste na compreensão em profundidade do processo de aliciamento de menores através do mundo digital, denominado grooming sexual online. Dos diversos objetivos específicos, destaca-se para a temática, o acesso ao procedimento de seleção das vítimas, a possíveis adaptações realizadas e ao modo de abordagem.

\section{PARTICIPANTES}

Na seleção dos participantes optou-se por "informantes privilegiados", tendo-se acedido a 4 indivíduos do sexo masculino, ofensores, 2 entrevistados e 2 envolvidos em diálogos sexuais com menores de idade, e a 1 vítima do sexo feminino, que foi entrevistada. Os conjuntos de diálogos online foram-nos cedidos pela vítima, tendo-se esta revelado uma fonte fundamental, por ter possibilitado 0 acesso a uma grande variabilidade de informação, decorrente do contacto envolvendo grooming sexual online com cerca de 25 ofensores. Todos os participantes eram maiores de idade na altura da recolha dos dados e foram selecionados através do Gabinete de Estudos e Atendimento a Agressores e Vítimas da Faculdade de Psicologia e Ciências de Educação da Universidade do Porto (GEAV).

\section{METODOLOGIA}

Face aos objetivos de investigação optou-se por uma metodologia qualitativa, sendo a recolha de dados realizada através de entrevista semiestruturada e análise documental de interações de teor sexual, ocorridas através de uma plataforma de comunicação online, entre ofensores e a respetiva vítima.

A análise dos dados, quer das entrevistas quer dos diálogos, foi realizada através de um procedimento de análise de conteúdo, como proposta por Bardin (2011), com construção de uma grelha de análise categorial do tipo semântico. 


\section{O ALICIAMENTO SEXUAL DE MENORES NA INTERNET: CONTRIBUTOS PARA O SEU CONHECIMENTO E PREVENÇÃO}

\section{RESULTADOS ALCANÇADOS}

Da totalidade de resultados encontrados destaca-se o surgimento dos grupos online como um modo de chegar a uma grande quantidade de jovens, ocorrendo a seleção dos menores a contactar através de critérios como a perceção de vulnerabilidade, detetada através das partilhas de informação pessoal que as vítimas realizam. Alguns perpetradores parecem adotar estratégias para se tornarem mais apelativos, com 0 intuito de aumentar a probabilidade de sucesso da abordagem, podendo realizar alterações no seu perfil ou na linguagem utilizada de modo a serem considerados mais atrativos.

Emergiram duas abordagens distintas dos menores, uma em que ocorria a introdução quase imediata de temáticas sexuais, que classificamos como direta, e outra em que o sujeito adota um conjunto de estratégias que têm por base a criação e manutenção de um vínculo relacional, que classificamos como indireta. Nesta última, o ofensor vai demonstrando interesse e disponibilidade para conhecer o menor e manter o contacto, à medida que se vai afirmando como uma figura de suporte emocional. As vulnerabilidades vão sendo exploradas, com 0 sujeito a tentar preencher as carências que vai encontrando. 0 laço afetivo desenvolvido vai sendo utilizado para manter a vítima na relação e conseguir a sua colaboração nos pedidos realizados que vão assumindo um carácter sexualizado. A utilização do material com conteúdo sexual anteriormente partilhado pelo menor é também utilizado para o coagir a manter-se cooperativo, estando esta estratégia presente em ambas as abordagens.

0 pedido de materiais como fotografias e vídeos, pode, inicialmente, não transparecer um carácter sexual, tornando-se este, numa fase posterior, explícito. Observou-se, ainda, o envolvimento das vítimas em grupos de partilha de conteúdos de cariz sexual.

Enquanto a finalidade de alguns sujeitos é exclusivamente o contacto online e as trocas associadas, para outros, este parece surgir como um meio para atingir o contacto pessoal, sendo esta pretensão expressa ao longo da comunicação.

\section{DISCUSSÃO}

Tal como referido em investigações anteriores, no nosso estudo foi possível verificar que os ofensores podem ter objetivos diferentes quando estabelecem contacto online com os menores e que, previamente ao contacto estabelecido, pode existir um processo de seleção onde sobressai a vulnerabilidade percebida (Briggs, Simon, \& Simonsen, 2010; Kloess et al., 2015; Webster et al., 2012). Observou-se, ainda, a possibilidade de ocorrerem modificações que revelam ter como propósito tornar o sujeito mais apelativo e aumentar a probabilidade de sucesso no estabelecimento de contactos, sendo a sua realização variável.

A diferenciação entre abordagem direta e indireta encontrada é consonante com os resultados de pesquisas anteriores (Kloess et al., 2017; Webster et al., 2012; Wolak et al., 2004), sendo que, se considerarmos o grooming como uma fase preparatória para 0 abuso, em que o desenvolvimento de um laço relacional é fundamental para as dinâmicas desenvolvidas durante a interação, apenas a abordagem que classificamos como indireta corresponde a este processo. Enquanto na interação indireta, o sujeito recorre ao vínculo relacional para conseguir o envolvimento da criança/jovem, explorando e aproveitando-se das suas vulnerabilidades, na interação mais direta o ofensor parece tirar proveito da curiosidade sexual típica do período de desenvolvimento. Destaca-se na abordagem indireta, a exploração das vulnerabilidades existentes e a colmatação destas pelo sujeito, sendo que esta é uma das estratégias que contribuem para o envolvimento do menor.

No decorrer dos contactos, para além das conversas sexualizadas e partilhas de conteúdos, pode ainda ocorrer a inserção da vítima em grupos de partilha de conteúdos sexuais, em que ocor- 
re a distribuição de pornografia de menores, sendo esta mais uma das múltiplas formas de vitimização associadas ao fenómeno.

\section{CONCLUSÕES}

0 aliciamento sexual de menores online constitui uma forma de abuso sexual, sendo uma realidade cada vez mais presente na atualidade devido à expansão e desenvolvimento do mundo digital e à maior presença das crianças e jovens neste mesmo.

A partilha diária de informação pessoal, sem restrições relativamente a quem pode aceder a esses mesmos conteúdos, permite que estes sujeitos se informem sobre os interesses dos menores e percecionem as suas vulnerabilidades, tornando-se mais cativantes.

0 fenómeno é complexo e diversificado, sendo o seu estudo fundamental para a informação e formação dos menores e dos adultos responsáveis e o desenvolvimento de medidas preventivas, tais como estratégias de supervisão parental e programas de prevenção nas escolas.

\section{REFERÊNCIAS BIBLIOGRÁFICAS}

Bardin, L. (2011). Análise de Conteúdo. Lisboa: Edições 70, Lda

Berson, I. R. (2003). Grooming Cybervictims. Journal of School Violence, 2(1), 5-18, doi:10.1300/J202v02n01_02

Black, P. J., Wollis, M., Woodworth, M., \& Hancock, J. T. (2015). A linguistic analysis of grooming strategies of online child sex offenders: Implications for our understanding of predatory sexual behavior in an increasingly computer-mediated world. Child Abuse \& Neglect, 1-10. http://dx.doi.org/10.1016/j.chiabu.2014.12.004

Branca, C. M., Grangeia, H., \& Cruz, 0. (2016). Grooming online em Portugal: Um estudo exploratório. Análise Psicológica, 34(3), 249-263. doi:10.14417/ap.978

Briggs, P., Simon, W. T., \& Simonsen, S. (2010). An Exploratory Study of Internet-Initiated Sexual Offenses and the Chat Room Sex Offender: Has the Internet Enabled a New Typology of Sex Offender? Sexual Abuse: A Journal of Research and Treatment, 20(10), 1-20. doi:10.1177/1079063210384275

Craven, S., Brown, S., \& Gilchrist, E. (2006). Sexual grooming of children: Review of literature and theoretical considerations. Journal of Sexual Aggression, 12 (3), pp. 287-299. doi:10.1080/13552600601069414

Davidson, J., Grove-Hills, J., Bifulco, A., Gottschalk, P., Caretti, V., Pham, T., \& Webster, S. (2011). Online abuse: Literature review and policy context. (Project Report) European online grooming project. Retirado de http://www.scotcen.org.uk/media/22523/european-online-grooming-projectliteraturereview.pdf

Kloess, J. A., Hamilton-Giachritsis, C. E., \& Beech, A. R. (2017). Offense Processes of Online Sexual Grooming and Abuse of Children Via Internet Communication Platforms. Sexual Abuse 00(0), 124, doi: 10.1177/1079063217720927

Kloess, J. A., Seymour-Smith, S., Hamilton-Giachritsis, C. E., Long, M. L., Shipley, D., \& Beech, A. R. (2015). A Qualitative Analysis of Offenders' Modus Operandi in Sexually Exploitative Interactions With Children Online. Sexual Abuse: A Journal of Research and Treatment, 29(6), 1-29. doi: $10.1177 / 1079063215612442$

Malesky, L. J. (2007). Predatory online behavior: modus operandi of convicted sex offenders in identifying potential victims and contacting minors over the internet. Journal Of Child Sexual Abuse, 16(2), 23-32. doi:10.1300/J070v16n02_02

McAlinden, A. M. (2006). "Setting 'em up": Personal, familial and institutional grooming in the sexual abuse of children. Social and Legal Studies, 15, 339-362. doi:10.1177/0964663906066613 


\section{O ALICIAMENTO SEXUAL DE MENORES NA INTERNET: CONTRIBUTOS PARA O SEU CONHECIMENTO E PREVENÇÃO}

United Nations Children's Fund. (2017). The State of the World's Children 2017: Children in a Digital World. Retirado de https://www.unicef.org/publications/index_101992.html

Wachs, S., Wolf, K. D., \& Pan, C. (2012). Cybergrooming: Risk factors, coping strategies and associations with cyberbullying. Psicothema, 24(4), 628-633

Webster, S., Davidson, J., Bifulco, A., Gottschalk, P., Caretti, V., Pham, T. ... Grove-Hills, J. (2012). European Online Grooming Project Final Report. European Union. http://www.european-onlinegrooming-project.com/

Winters, G. M., \& Jeglic, E. L. (2016). Stages of Sexual Grooming: Recognizing Potentially Predatory Behaviors of Child Molesters. Deviant Behavior, 1-10, doi: 10.1080/01639625.2016.1197656

Wolak, J., Finkelhor, D., \& Mitchell, K. (2004). Internet-initiated Sex Crimes against Minors: Implications for Prevention Based on Findings from a National Study. Journal of Adolescent Health, 35(5), 11-20. doi:10.1016/j.jadohealth.2004.05.006 